\section{Low body mass index can be associated with the risk and poor outcomes of neuromyelitis optica with aquaporin-4 immunoglobulin $\mathrm{G}$ in women}

\section{INTRODUCTION}

Neuromyelitis optica spectrum disorder with aquaporin-4 immunoglobulin G (NMOSD-AQP4) is an inflammatory disease of the central nervous system (CNS). Recent studies have demonstrated that body mass index (BMI) can be associated with the risk and/or outcomes of diverse inflammatory diseases, such as rheumatoid arthritis (RA), psoriasis, Hashimoto's thyroiditis and multiple sclerosis (MS). However, the clinical implication of BMI in NMOSD-AQP4 is still unknown.

\section{MATERIALS AND METHODS Patients}

A total of 157 patients with NMOSD-AQP4 who had their first episodes between 1982 and 2016, from six nationwide tertiary referral hospitals, were finally included (see figure 1 in the online Supplementary file 1). Patients were tested for antiaquaporin-4 (AQP4) antibody using either the fluorescence-activated cell sorting assay or cell-based assay. ${ }^{1}$ In the NMOSD-AQP4 group, BMI measured within 1 month of the first symptom of NMOSD-AQP4 and before any steroid treatment, immune suppressant, or immune modifying treatment $\left(\mathrm{BMI}_{\text {naive }}\right)$ and the lowest BMI measured within 1 year of the first symptoms of NMOSD-AQP4 (BMI ${ }_{\text {min-1yr }}$ ) were obtained, respectively. BMI values less than $18.5 \mathrm{~kg} / \mathrm{m}^{2}$ were classified as low according to the WHO recommendation. Demographic information, number and location of attacks, results of the cerebrospinal fluid examination and maximal score of the Kurtzke Expanded Disability Status Scale (EDSS) on disease onset were assessed. Prognosis was evaluated in terms of the time to a confirmed EDSS of 6 (EDSS6), functional system score of 4 for visual function (VF4) and mortality.

\section{Healthy controls and MS controls}

A matched case-control study was performed to evaluate the association between BMI and risk for NMOSD-AQP4. The controls were selected from the 96146 participants included in the nationwide population-based survey of the health and nutritional status of Korea (Korean National Health and Nutrition Examination Survey (KNHANES)) ${ }^{2}$ conducted in 1998, 2001, 2005 and 2007-2015. Four controls were matched to each case based on age, sex and year of BMI measurement (within a maximum of 3 years). Seventy-five patients with NMOSD-AQP4 who have available $\mathrm{BMI}_{\text {naive }}$ data were matched after excluding those who were diagnosed before $1995(n=3)$ and those with missing BMI information on diagnosis $(n=79)$. The BMI of the 300 controls and BMI of the 75 patients with NMOSD-AQP4 were finally included in the matched casecontrol study.

Sixty-three patients who met the international panel criteria for MS from four tertiary hospitals were included in the MS control group. Demographic data and clinical information, including $\mathrm{BMI}_{\text {naive }}$ and $\mathrm{BMI}_{\text {min-1yr }}$, were obtained. Among them, 33 had the BMI naive $_{\text {mata and were }}$ diagnosed since 1995.

The demographic data and clinical characteristics of the NMOSD-AQP4 and MS cohort are shown in table 1 in the online Supplementary file 2 .

\section{Statistical analysis}

Conditional logistic regression analysis was performed using the matched data of the BMI naive $_{\text {of individuals with }}$ NMOSD-AQP4 and BMI of healthy controls to evaluate the association of BMI with the risk of NMOSD-AQP4. A multivariable binary logistic regression analysis was performed for comparing the BMI of individuals with NMOSD-AQP4 on the onset of symptoms with that of the MS controls. The association between BMI and disability on the onset of NMOSD-AQP4 was analysed using a multivariable linear regression between $\mathrm{BMI}_{\text {naive }}$ and EDSS at nadir of the first NMOSD-AQP4 attack.

The prognostic values of $\mathrm{BMI}$ in patients with NMOSD-AQP4 were also tested using univariable and multivariable Cox proportional hazards regression with Firth's penalised likelihood bias-reduction method because of low events and convergence failures in the Cox regression. A P value of $<0.05$ was considered statistically significant. Statistical analyses were performed using SPSS software V.22.0 and SAS V.9.2.

\section{RESULTS}

$\mathrm{BMI}$ and the risk for NMOSD-AQP4

Based on the conditional logistic regression analysis, a lower BMI was significantly associated with the risk for NMOSD-AQP4 among women (OR 1.17 per one unit decrease; 95\% CI 1.06 to 1.28 ). However, no association was observed between BMI and the risk for NMOSD-AQP4 in men (OR 1.02 per one unit decrease; 95\% CI 0.83 to 1.24; table 1). Sex and age were not confounded with disease in case-control matching data based on sex and age $(\mathrm{P}=0.888$ and $\mathrm{P}=0.455$, respectively; see table 2 in the online Supplementary file 2).

Based on a multivariable binary logistic regression analysis with age as covariates, a lower BMI ${ }_{\text {naive }}$ was significantly associated with the risk of developing NMOSD-AQP4 compared with MS among women (OR 1.20 per one unit decrease; 95\% CI 1.00 to $1.14, \mathrm{P}=0.046$; table 2 ). However, no association was observed between $\mathrm{BMI}$ and NMOSD-AQP4 and MS in men. The demographic data and clinical characteristics of individuals with NMOSD-AQP4 and MS who have available BMI naive $_{\text {data }}$ are shown in table 3 in the online Supplementary file 2 .

Table 1 The association of BMI with the risk and outcomes of NMOSD-AQP4

\begin{tabular}{|c|c|c|c|c|}
\hline & \multicolumn{2}{|l|}{ Women } & \multicolumn{2}{|l|}{ Men } \\
\hline & NMOSD-AQP4* $(n=64)$ & Healthy controls $(n=256)$ & NMOSD-AQP4* $(n=11)$ & Healthy controls $(n=44)$ \\
\hline $\mathrm{BMI}$, mean (SD), $\mathrm{kg} / \mathrm{m}^{2}$ & $21.4(2.9)$ & $23(3.6)$ & $23.4(2.7)$ & $23.6(3.8)$ \\
\hline OR $(95 \% \mathrm{Cl})$ & 1.17 (1.06 to 1.28$)$ & & 1.02 (0.83 to 1.24$)$ & \\
\hline$P$ value & 0.001 & & 0.865 & \\
\hline
\end{tabular}

\footnotetext{
*The BMI of NMOSD-AQP4 were BMI ${ }_{\text {naive }}$ which is measured within 1 month of their disease onset and measured before the use of steroid treatment.
}

BMI, body mass index; NMOSD-AQP4, neuromyelitis optica spectrum disorder with aquaporin-4 immunoglobulin G. 
Table 2 Binary logistic regression analysis of the risk factors for NMOSD-AQP4 compared with MS

\begin{tabular}{|c|c|c|c|c|}
\hline & \multicolumn{2}{|l|}{ Women } & \multicolumn{2}{|l|}{ Men } \\
\hline & $\mathrm{OR}^{*}(95 \% \mathrm{Cl})$ & $P$ value & $\mathrm{OR}^{*}(95 \% \mathrm{Cl})$ & $P$ value \\
\hline $\begin{array}{l}\mathrm{BMI}_{\text {naive' }} \text { one unit } \\
\text { decrease }\end{array}$ & 1.20 (1.00 to 1.43$)$ & 0.046 & $0.8(0.47$ to 1.28$)$ & 0.338 \\
\hline $\begin{array}{l}\text { Age at onset } \\
(\text { ref: }<20)\end{array}$ & 1 (reference) & - & 1 (reference) & - \\
\hline $20-29$ & $1.09(0.22$ to 5.40$)$ & 0.135 & $0.05(<0.001$ to 4.50$)$ & 0.201 \\
\hline $30-39$ & $1.3(0.28$ to 6.05$)$ & 0.214 & $0.13(0.002$ to 4.37$)$ & 0.253 \\
\hline $40-49$ & 3.37 (0.58 to 19.63$)$ & 0.552 & 0.33 (0.002 to 31.18$)$ & 0.621 \\
\hline $50-$ & $\begin{array}{l}15.67 \text { (1.44 to } \\
171.12)\end{array}$ & 0.031 & $\begin{array}{l}3.42(0.057 \text { to } \\
839.73)\end{array}$ & 0.558 \\
\hline
\end{tabular}

${ }^{*}$ Binary logistic regression (reference: MS). Control variables included BMI, age at onset (categorised every 10 years). BMI $_{\text {naive' }}$ which is measured within 1 month of their disease onset and measured before the use of steroid treatment.

$\mathrm{BMI}_{\text {naver }}$ body mass index is measured within 1 month of their disease onset and measured before the use of steroid treatment; NMOSD-AQP4, neuromyelitis optica spectrum disorder with aquaporin-4 immunoglobulin G.

BMI and outcomes of NMOSD-AQP4 On the onset of NMOSD-AQP4, BMI was negatively correlated with nadir EDSS in women with NMOSD-AQP4, although no statistical significance was observed $(\beta=-0.463 ; P=0.065$; see table 4 in the online Supplementary file 2).

The Kaplan-Meier survival analysis showed that a low BMI $I_{\text {min-1yr }}\left(<18.5 \mathrm{~kg} / \mathrm{m}^{2}\right)$ was a significant predictor of long-term mortality in women with NMOSD-AQP4 $(\mathrm{P}=0.001$ via $\log$-rank test). However, a low $\mathrm{BMI}_{\text {nave }}$ did not predict the prognosis of women with NMOSD-AQP4 (see figure 2 in the online Supplementary file 3). An analysis of the relationship between BMI and prognosis in men with NMOSD-AQP4 could not be performed because of the limited number of participant (five with EDSS6, five with VF4 and one mortality). The demographic data and clinical characteristics of patients with low BMI min-1yr $_{1}\left(<18.5 \mathrm{~kg} / \mathrm{m}^{2}\right)$ and normal $\mathrm{BMI}_{\text {min-1yr }} \stackrel{\text { min-1yr }}{\left(\geq 18.5 \mathrm{~kg} / \mathrm{m}^{2}\right)}$ are shown in table 5 in the online Supplementary file 2.

Multivariable Cox proportional hazards regression with age at onset of disease and use of immunosuppressants and steroids as covariates revealed that low $\mathrm{BMI}_{\text {min-1yr }}$ was considered as a significant predictor of long-term mortality in women with NMOSD-AQP4 (table 3).

\section{DISCUSSION}

In the present study, a lower BMI was associated with the risk for NMOSD using matched data in women with NMOSD-AQP4 and healthy controls. Moreover, lower BMI was also associated with the risk for NMOSD-AQP4 compared with MS among women. Lastly, a low BMI $_{\text {min-1yr }}$ was a predictor of mortality, which is independent of age at onset, the use of oral steroids or immunosuppressants in women with NMOSD-AQP4. All these findings suggested that a low BMI could be a risk factor of NMOSD-AQP4 in women.

In numerous studies, the association between BMI and the risk and/or outcomes of diverse autoimmune diseases has been reported. A low BMI in RA (rheumatoid cachexia) has been consistently found to be associated with high disease activities and mortality. ${ }^{3}$ Since NMOSD-AQP4 and systemic rheumatological disease can share some susceptibilities to common autoimmunity, the association between low BMI and the onset and poor outcomes of NMOSD-AQP4 is supported by the previous studies on RA.

We hypothesized that one of the following mechanisms may be responsible. First, a low BMI could increase the susceptibility to autoimmunity due to the deregulated level of adipokines ${ }^{4}$ or sex hormones. ${ }^{5}$ Second, some common predisposing factors (per se, genetic or environmental factors) could exist that might affect both BMI and the pathogenesis of NMOSD-AQP4. Lastly, low BMI might be the prodromal symptoms of NMOSD, rather than the cause of the disease.

Furthermore, our results suggested that BMI might play a different role in NMOSD-AQP4 and MS since obesity has been associated with an increased risk of developing MS. ${ }^{6}$ This difference is most likely attributed to the distinct pathogenesis, immunology and comorbidities of these two diseases, despite some minor phenotypical overlaps. Moreover, as our study showed that among women the risk of NMOSD-AQP4 compared to MS increased with the lower BMI ${ }_{\text {naive }}$ that was measured before use of any steroid, immune suppressants, or immune-modifying treatment, this phenomenon semms to be independent of the different treatment between those two diseases.

In our study, the low BMI in the earlier stage of disease $\left(\mathrm{BMI}_{\text {min-1yr }}\right)$ was a predictor for mortality with a HR of 19.26 among women in a multivariable analysis with age of onset, use of steroid and immune suppressant. This finding implies that BMI in the earlier stage of the disease might be a more potent predictive factor for mortality than previously known ones (per se, the age of onset or types of treatment).

Our study revealed that low BMI was associated with the onset and outcomes of NMOSD-AQP4 only in women. These results might be attributable to sex-dependent immune response (per se, the distinct expression of the sex hormonebinding globulin). Nevertheless, the limited number of male patients with

Table 3 Multivariable Cox proportional hazards regression to determine predictors for the long-term mortality in women with NMOSD-AQP4

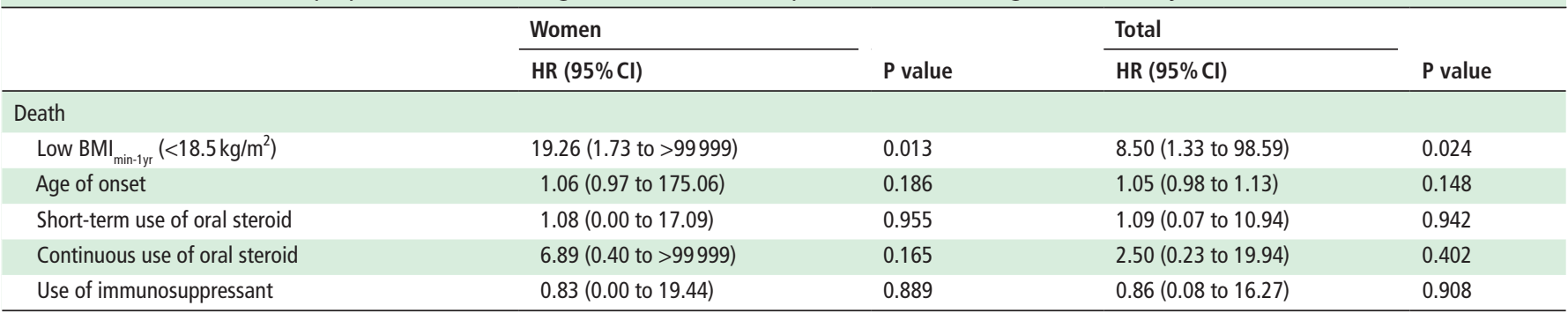

$\mathrm{BMI}_{\text {min-1yt }}$ the lowest body mass index measured within 1 year of the first symptoms;NMOSD-AQP4, neuromyelitis optica spectrum disorder with aquaporin-4 immunoglobulin G. 
NMOSD-AQP4 in our study could be responsible for it.

In conclusion, a low BMI can be a risk factor for NMOSD-AQP4 in women, as it was associated with the risk of disease and higher mortality in women with NMOSD-AQP4.

Seol-Hee Baek, ${ }^{1,2}$ Ji-Sun Kim, ${ }^{2}$ Myoung-jin Jang, ${ }^{3}$ Yoo Hwan Kim, ${ }^{2,4}$ Ohyun Kwon, Jung-Hwan $\mathrm{Oh}^{6}{ }^{6}$ Sa-Yoon Kang, ${ }^{6}$ Ji-Hoon Kang, ${ }^{6}$ Kee Hong Park, ${ }^{7}$ Yong-Shik Park, ${ }^{8}$ Kyung Seok Park, ${ }^{8}$ Dong Wook Shin, ${ }^{9}$ Byung-Jo Kim, ${ }^{2}$ Sung-Min Kim ${ }^{1}$

1 Department of Neurology, Seoul National University Hospital, Seoul, Korea

${ }^{2}$ Department of Neurology, Korea University Medical Center, Korea University College of Medicine, Seoul, Korea

${ }^{3}$ Medical Research Collaborating Center, Seoul National University Hospital, Seoul, Korea

${ }^{4}$ Department of Neurology, Hangang Sacred Heart Hospital, Hallym University Medical Center, Seoul, Korea ${ }^{5}$ Department of Neurology, Eulji University College of Medicine, Seoul, Korea

${ }^{6}$ Department of Neurology, Jeju National University School of Medicine, Jeju, Korea

${ }^{7}$ Department of Neurology, Gyeongsang National University Hospital, Jinju, Korea

${ }^{8}$ Department of Neurology, Seoul National University Bundang Hospital, Seongnam, Gyeonggi-do, Korea ${ }^{9}$ Department of Family Medicine and Supportive Care Center, Samsung Medical Center, Seoul, Korea

Correspondence to Professor Sung-Min Kim, Department of Neurology, Seoul National University Hospital, Seoul 03080, Republic of Korea; sueh916@ gmail.com

Contributors SHB: acquisition and analysis of data, statistical analysis and drafting of the manuscript. MJJ: statistical analysis. JSK, YHK, OK, JHO, SYK, JHK, KHP, YSP and KSP: acquisition and analysis of data. DWS: analysis of data. BJK: study concept and design, acquisition and analysis of data and critical revision of manuscript. SMK: study concept and design, acquisition and analysis of data, critical revision of manuscript and drafting of the manuscript. * SMK and BJK contributed equally to this paper.

Funding This study was supported by Grant No. 2017R1D1A1B03031490 from the National Research Foundation of Korea.

Competing interests None declared.

Ethics approval This study was approved by the Institutional Review Board (IRB) of Seoul National University Hospital (IRB number: H-1609-084-792).

Provenance and peer review Not commissioned; externally peer reviewed.

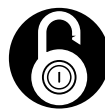

\section{OPEN ACCESS}

Open access This is an open access article distributed in accordance with the Creative Commons Attribution Non Commercial (CC BY-NC 4.0) license, which permits others to distribute, remix, adapt, build upon this work non-commercially, and license their derivative works on different terms, provided the original work is properly cited and the use is non-commercial. See: http:// creativecommons.org/licenses/by-nc/4.0/

(c) Article author(s) (or their employer(s) unless otherwise stated in the text of the article) 2018. All rights reserved. No commercial use is permitted unless otherwise expressly granted.

- Additional material is published online only. To view please visit the journal online (http://dx.doi.org/ 10.1136/jnnp-2017-317202).

BJK and SMK contributed equally.

\section{(D) Check for updates}

To cite Baek S-H, Kim J-S, Jang M, et al. J Neurol Neurosurg Psychiatry 2018;89:1228-1230.

Received 6 September 2017

Revised 22 November 2017

Accepted 1 January 2018

Published Online First 25 January 2018

J Neurol Neurosurg Psychiatry 2018;89:1228-1230. doi:10.1136/jnnp-2017-317202

\section{REFERENCES}

1 Wingerchuk DM, Banwell B, Bennett JL, et al. International consensus diagnostic criteria for neuromyelitis optica spectrum disorders. Neurology 2015;85:177-89.

2 Korea Centers for Disease Control and Prevention. Korean National Health and Nutrition Examination Survey. https://knhanes.cdc.go.kr/knhanes

3 Wolfe F, Michaud K. Effect of body mass index on mortality and clinical status in rheumatoid arthritis. Arthritis Care Res 2012:64:1471-9.

4 Ouchi N, Parker JL, Lugus JJ, et al. Adipokines in inflammation and metabolic disease. Nat Rev Immunol 2011:11:85-97.

5 Ansar Ahmed S, Penhale WJ, Talal N. Sex hormones, immune responses, and autoimmune diseases. Mechanisms of sex hormone action. Am J Pathol 1985:121:531-82.

6 Munger KL, Chitnis T, Ascherio A. Body size and risk of MS in two cohorts of US women. Neurology 2009;73:1543-50. 